\title{
THICKNESS DIFFERENCE OF ELECTRODE COATINGS CAUSED BY ELASTIC TURBULENCE OF ELECTRODE COMPOUNDS UNDER CONDITION OF NONISOTHERMAL PRESSURE FLOW
}

\author{
A.E. MARCHENKO \\ E.O. Paton Electric Welding Institute, NASU \\ 11 Bozhenko Str., 03680, Kiev, Ukraine. E-mail: office@paton.kiev.ua
}

\begin{abstract}
Generalized and analyzed are the results of investigations carried at the E.O. Paton Electric Welding Institute on technological properties of electrode compounds, promoting thickness difference of electrode coatings. The investigations were carried out using capillary viscosimeter of fixed flow - rheometer with electric drive, which can be used for simulation of the conditions of compound extrusion application over the electrode rods, typical for commercial electric extrusion presses. Thus, specific pressure on compound under condition of stationary flow achieves 60-75 MPa, average gradient of shear rate is gradually regulated in the ranges from 1 to $5 \cdot 10^{3} \mathrm{~s}^{-1}$, and it makes to $65 \cdot 10^{3} \mathrm{~s}^{-1}$ for separate types of compound. The compounds for rutile, low-hydrogen and cellulose electrodes, differing by wide range of consistency indices, were investigated. In addition to shear and longitudinal viscosity, the investigations were carried out on a range of change of their modulus of shear elasticity, period of relaxation, criterion of Reynolds elastic turbulence, elastic potential and reversible (elastic) deformation. The results received were analyzed from point of view of existing phenomenological theory of elastic turbulence of polymer materials, combining viscosity and elasticity properties. It is successfully used in the course of many years in rheology of melts and solutions of high-molecular compounds for solving of technological problems of their extrusion processing. Variants of elastic turbulence, detected in capillary and pre-capillary zones, were analyzed. Quality relation of this phenomenon to appearance and nature of coating thickness difference in course of real extrusion application of compound over the electrode rods was shown. 24 Ref., 4 Tables, 9 Figures.
\end{abstract}

Key words: arc welding, coated electrodes, coating thickness difference, electrode compounds, viscosity, elasticity modulus, elastic turbulence

Alignment of coating casing and rod [1,2] is one of the most important among number of indices of GOST 9466-75, which are used by customer for assessment of quality of the electrode production. Thickness difference prevents normal welding process as well as results in deterioration of quality and mechanical properties of the welds. Thickness difference of the coating, which is maximum allowable by standard, i.e. not exceeding $5 \%$ of the rod diameter, and, moreover, one which is above the norm, promotes formation one-side «peak» at the end of consumable electrode that disrupts air protecting gas and slag coverage of molten metal during welding, as well as formation of weld metal. This results in unfavorable changes of chemical composition, deterioration of mechanical properties, formation of pores and other weld defects [3-5].

It is believed that number of factors result in appearance of thickness difference of the coating (type, portion and grain composition of constitu- ents, technological characteristics of compounds, quality of rods, condition of equipment, qualification and level of discipline of staff, engaged in manufacture of electrodes [6]), and they prevent finding the real reasons and regularities of its appearance in course of many years. This limited the capabilities of accurate prediction of quality of the electrodes on this index.

Compound is applied over steel rods by means of extrusion. This process should be termed in such a way, since change of shape in order to transform the compound in concentric circular casing around the rod is preceded by its all-round compression in a head of extrusion press. It is the key operation in technology of production of welding electrodes, which, in particular, results in coating thickness difference.

Experience, gained in other technological processes, using extrusion method for processing of paste, including filled materials, similar on consistency to the electrode compounds, shows that interruption of their stable and uniform flowing from the shaping instrument is determined by the following main reasons [7]:

- slipping of flow near the walls of shaping instrument; 
- breaking of elastic liquids under effect of huge stresses;

- elastic hydrodynamic instability, accompanied by increasing disturbances (it is called elastic turbulence);

- structural instability, caused by viscosity abnormity (appearing, in particular, in form of its reduction under effect of increase of rate gradient and temperature).

Studies of rheological properties of the electrode compounds, carried by the E.O. Paton Electric Welding Institute in course of many years, showed that elastic turbulence is considered as the main reason of appearance of coating thickness difference. The rest can intensify it, whereas flow interruptions under effect of huge stress can be observed extremely rare.

Elastic hydrodynamic instability takes place in the electrode compounds, combining viscous and elastic properties, relationship between which is changed during their extrusion treatment. Excess of elasticity can promote regular (oscillatory) or irregular disturbances in the flow. Viscosity can be a damping constituent of the rheological system, i.e. suppress elastic constituent. If viscosity and elasticity modulus are equal, then loss of electrode compound stable flow takes place. Point, where it happens, can be the surface of gage sleeve and other sections of the shaping instrument. Time, when it happens, depends on the fact when compound viscosity is reduced due to temperature and/or structural breaking of the coagulation structure to such extent that capability to suppress flow pulses, promoted by elastic stresses, is lost. The higher elasticity in comparison with viscosity, the more is the difference of irregular disturbances from the regular form. In particular, if the system has more than one center of such disturbances. Aim of the present work is to show dependence between plastic turbulence of the electrode compound and thickness difference of electrode coating.

Rheological model of visco-elastic materials and mathematical formulae of their description. The simplest one-dimension rheological model, which can be used during investigation of properties of the electrode compounds, was proposed by Maxwell [8]. It is represented in form of spring (Hooke elastic body) connected in sequence with hydraulic damper (viscous Newtonian fluid). The model shows the reason of appearance and allows explaining the process of stress relaxation in visco-elastic material under constant deformation or stress. Visco-elastic liquid on Maxwell model, being subjected to the deformation promoted by constant force applied to it, should deform step-like by value of compression (extension) of the elastic element, and further deform with constant rate, corresponding to the applied force. If the same model is quickly deformed using the set value $\gamma$, and the force (or stress) change, proportional to the set deformation, is examined after fixing $\gamma$ on this level, then they will gladually reduce (relax) in time due to shear of damper piston. Mathematic law, describing rheological behavior of such model is the fowling:

$$
\dot{\gamma}=\frac{\tau}{\eta} \frac{1}{G} \dot{\tau},
$$

and process of stress relaxation under constant deformation

$$
\tau=\tau_{0} e^{(G / \eta) t} .
$$

Since, $\lim \underset{t \rightarrow \infty}{\tau}=\lim e_{t \rightarrow \infty}^{(G / \eta) t}=0$, then the stress $\tau$ under constant deformation in time will exponentially go to zero (here and below for designations see Table 1).

Relationship of dynamic viscosity $\eta$ to elasticity modulus $G$ has time dimension and is termed as a period of relaxation, $\tau_{1}=\theta$. Taking this into account

$$
t_{1}=\frac{\eta}{G} ; \quad \frac{\tau_{1}}{\tau_{0}}=e^{(G / \eta) t}=e^{-1}=0.37,
$$

and $\tau_{1}=0.37 \tau_{0}$.

Therefore, initial stress reduces by $63 \%$ per time $\theta$.

Combining the relaxation period $\theta$ and duration $t_{h}$ of the external influence on material can help to evaluate its behavior in change of deformation rate.

$\tau$ and $\eta$ are determined, first of all, in order to evaluate rheological behavior of the compound at its forced flow [9]. Then, such indices as $G$, $\theta$ and elastic potential $W$ are calculated using formulae, proposed and tested in the technology of extrusion processing of polymer materials. They together provide for complete and objective characteristic of visco-elastic indices of the compounds.

There are three methods for calculation of pressure drop on capillary length, $\Delta P$, necessary for calculation of $\tau$ and $\eta$ from general resistance of measuring cell as a constituent of capillary and pre-capillary (entrance) zone. These are methods of Couette, Bagley and lock disk.

Couette method, assuming that resistance of capillary to flow rises in proportion to its length, for calculation of shear stress on capillary wall applies a pressure difference, registered in use of 
long and short capillaries of the same diameter $\left(\Delta P=P_{\text {long }}-P_{\text {short }}\right)$.

In Bagley method [10] the dependence of pressure difference on specific length of channel, $L / R$, at fixed shear rate was also assumed linear, and $\Delta P_{0}$ value is calculated by means of extrapolation of $\Delta P=f(L / R)$ dependence to $L / R$ zero value. The extrapolation of the same dependence for zero pressure value gives the value of dummy channel extension, $n_{\mathrm{B}}$, equivalent to input pressure loss on resistance. It is called Bagley correction.

Couettee and Bagley methods cannot always be used in rheological testing of the electrode compounds, since pressure loss in their flowing through capillaries is not always proportional to their given length, $L / d_{\mathrm{c}}$.

On the one hand, it is caused by accumulation of elastic stresses in initial sections of the short capillaries; on the other one, it is promoted by excessive dissipative heating of the compound jet in near-wall layer in use of capillaries of excessive length. The first and the second do not allow determining the slope of line $\Delta P=f\left(L / d_{\mathrm{c}}\right)$ with accuracy necessary for $\tau$ calculation. Therefore, lock disk was used for determination of $P_{0}$ and $\triangle P$ was calculated as $\left(P_{L}-P_{0}\right)$ difference at $L=$ $=10 d_{\mathrm{c}}=20 R$. In our case such a procedure provides for the highest accuracy of the test results.

At the same time, by example of the other authors, Bagley approach was used for reveal of rheological principle of correction $n_{\mathrm{B}}$, as well as for separation from it of the elastic constituent, necessary for $G$ calculation.

Rheological essence of Bagley correction can be determined using the following mathematical developments.

Shear stress on the capillary wall according to Couettee method, considering taken by us designations (see note to Table 1), is calculated on formulae

$$
\tau=\frac{P R}{2 L}=\frac{P}{2(L / R)}=\frac{P}{2 n_{\mathrm{C}}} .
$$

Taking into account Bagley correction the expression for calculation of $\tau$ from (4) is transformed into

$$
\begin{gathered}
\tau=\frac{P}{2\left(n_{\mathrm{C}}+n_{\mathrm{B}}\right)} ; \quad 2 \tau\left(n_{\mathrm{C}}+n_{\mathrm{B}}\right)=P ; \\
n_{\mathrm{B}}=\frac{P-2 \tau n_{\mathrm{C}}}{2 \tau}=\frac{P}{2 \tau}-n_{\mathrm{C}} .
\end{gathered}
$$

When $L=0$ (as in the case when input resistance is found by lock disk method), $n_{\mathrm{C}}=0, P=$ $=P_{0}, n_{\mathrm{B}}=P_{0} / 2 \tau$. Therefore, Bagley correction represent itself a value of input resistances, rated on shear stress on the capillary wall.

In such a form Bagley correction was used as a basis for evaluation of value of natural convergence angle, which is formed in pre-capillary zone at extrusion processing of visco-elastic materials [11-13].

It can be shown that in this meaning $n_{\mathrm{B}}$ correction is equivalent to criterion of Reynolds elastic turbulence, $\mathrm{Re}_{\mathrm{e}}$, or, as it was called earlier by Rainer, Deborah number (name of ancient pythoness ) [14, 15].

In general, this is a reversible elastic deformation of the visco-elastic material, $\gamma$, subjected to it under the effect of deformation stress. It was mention above that it is estimated as a relationship of period of relaxation to typical time, $\theta / t_{h}$. For capillary viscometer $t_{h}$ equals the value inverse to gradient of shear rate [14, 15] and, respectively, $\operatorname{Re}_{\mathrm{e}}=\theta \dot{\gamma}$.

Reynolds criteria is transformed in the same form in the following way:

$$
\operatorname{Re}_{\mathrm{e}}=\frac{\theta R}{U T^{2}}=\frac{\theta}{U T^{2} / R}=\frac{\theta}{\dot{\gamma} T^{2}}=\theta \dot{\gamma} .
$$

On the other hand, considering that $\theta=\eta / G$ and $G=\tau^{2} / P_{0}$, equation (6) can be equated to expression $P_{0} / \tau$ approving, in such a way, that Bagley input correction represents itself criterion of Reynolds elastic turbulence.

Assuming, as it proposed in work [16], that $n_{\mathrm{B}}$ in formula (5) together with Couettee correction includes elastic deformation $S_{R}$ as well, which is part of formula of Hooke's law $\tau=G S_{R}$, then $n_{\mathrm{B}}=n_{\mathrm{C}}+\left(S_{R} / 2\right)=n_{\mathrm{C}}+(1 / 2 G) \tau$. It follows that $\tau$ and $P_{0}$ value, determined by means of capillary measurements, can help to calculate modulus of shear elasticity $G$ of the electrode compounds.

In fact, at $n_{\mathrm{C}}=0$

$$
G=\tau / 2\left(n_{\mathrm{B}}\right)=\tau^{2} / P_{0} .
$$

Elastic potential $W$ is calculated on input resistances. It is elastic energy referred to consumption per second, accumulated by the compound during flow through capillary: $W=P_{0} / 12$. It is revealed at the output from capillary in form of free elastic recovery of jet, which is accompanied by deformation $-\beta$ times increase of its diameter and $\beta^{2}$ times of length. A balance of revealed and accumulated energy is described by relationship [8]

$$
W=\frac{G}{2}\left(\beta^{4}+2 \beta^{-2}-3\right),
$$


Table 1. Summary table of formulae used for calculation of rheological indices of compounds under condition of pressure flow through capillary of viscosimeter

\begin{tabular}{|c|c|}
\hline Index of viscoelastic material & Formula \\
\hline Shear viscosity, MPa·s & $\eta=\tau / \dot{\gamma}$ \\
\hline Longitudinal viscosity, MPa.s & $\lambda=\frac{9\left(n_{0}+1\right)^{2}}{32 \eta}\left(\frac{P_{0}}{\dot{\gamma}}\right)^{2}$ \\
\hline Modulus of shear elasticity, MPa & $G=\eta / \theta=\tau^{2} / P_{0}$ \\
\hline Angle of natural convergence, deg & $\operatorname{tg} \alpha_{0}=\sqrt{2 \eta / \lambda}$ \\
\hline Characteristic Maxwell relationship. Dimensionless time of relaxation & $n_{\mathrm{B}}=\theta / t_{h}=\theta \dot{\gamma}=P_{0} / 2 \tau=\operatorname{Re}_{\mathrm{e}}$ \\
\hline Elastic potential, MPa & $W=\tau^{2} / 6 G=P_{0} / 12$ \\
\hline Reversible (elastic) deformation & $\gamma=\tau / G=\sqrt{3\left(\beta^{4}+2 \beta^{-2}-3\right)}$ \\
\hline \multicolumn{2}{|c|}{$\begin{array}{l}\text { Here, } \tau-\text { stress on capillary wall, MPa; } \dot{\gamma}-\text { average gradient of shear rate, } \mathrm{s}^{-1} ; n_{0}-\text { flow index equal } \lg \tau / \lg \dot{\gamma} ; P_{0}-\text { losses of flow in } \\
\text { entrance zone, MPa; } G-\text { modulus of shear elasticity, MPa; } \theta-\text { period of relaxation, s; } \alpha_{0}-\text { angle of natural convergence, deg; } \gamma- \\
\text { reversible elastic deformation of jet; } t_{h}-\text { characteristic time of monitoring, in capillary viscosimetry, } t_{h}=1 / \dot{\gamma} ; W-\text { elastic potential, } \\
\text { specific elastic energy, accumulated by compound in flowing through capillary, in calculation per unit of its volume (consumption per } \\
\text { second } Q) ; \beta, \beta^{2}-\text { level of expansion of compound jet at the output from capillary on diameter and length, respectively, rel. un. }\end{array}$} \\
\hline
\end{tabular}

and value of deformation, promoted by jet swelling - by relationship

$$
\gamma=\sqrt{3\left(\beta^{4}+2 \beta^{-2}-3\right)} .
$$

In order to make it simple in use, received formulas are given in Table 1.

Electrode compounds as pasty compositions having visco-elastic properties. There is sufficient number of publications, indicating that electrode compounds together with viscosity should actually have elasticity properties.

Thus, author of work [17] characterizes plasticity of compounds by such rheological coefficients as shear elasticity and viscosity, and curve of its flowing is represented in «pressure $P-$ consumption per second $Q \gg$ variables. At low consumption it is differ from classical Bingham rheogram, describing plastically-viscous body, termed as a consequence by his name, by power splash of pressure, which at $Q_{\mathrm{cr}} \approx 1 \mathrm{~cm}^{3} \cdot \mathrm{s}^{-1}$ smoothly transfers into line, slightly inclined to consumption axis. The reasons of maximum appearance had no explanations. We assumed that it is a result of relaxation of accumulated elastic stresses.

Thesis in [18] describes a procedure for determination of mentioned above indices of elasticity and viscosity of the electrode compounds. For this, the compound was slowly pressed by plunger through capillary sectioned on length. Flow pressure and volume of compound, escaping from the capillary under effect of elastic forces, were registered two times (during plunger stop and after switching off at stationary plunger in capillary end section). Indices of shear elasticity and viscosity, calculated using obtained data, are given in Table 2.

Our investigations of ANO-4 compound, pressed between two corrugated plates, also carried out in modes of shear creep flow, showed that it, the same as other types of concentrated dispersions of particles with crystalline structure, is significantly strengthened under the effect of shear stresses of around 0.01 MPa value. Initial deformation capacity of the compound in repeated loading is reproduced only after 4 hours

Table 2. Technological properties of compounds TsM-7 and UONI-13/55 in mode of creep pressure flow [18]

\begin{tabular}{|c|c|c|c|c|c|c|}
\hline \multicolumn{3}{|c|}{ Liquid glass } & \multirow{2}{*}{$\begin{array}{c}\text { Portion of } \mathrm{Na}_{2} \mathrm{CO}_{3} \text {, } \\
\%\end{array}$} & \multirow{2}{*}{$P, \mathrm{MPa}$} & \multirow{2}{*}{$G, \mathrm{MPa}$} & \multirow{2}{*}{$\eta \cdot 10^{-6}, \mathrm{mPa} \cdot \mathrm{s}$} \\
\hline Modulus & Density, $\mathrm{kg} / \mathrm{m}^{3}$ & Portion, wt.\% & & & & \\
\hline \multicolumn{7}{|c|}{ Compound of electrodes TsM-7 } \\
\hline \multirow[t]{2}{*}{$\mathrm{Na}_{2} \mathrm{O}_{2} \cdot 8 \mathrm{SiO}_{2}$} & \multirow[t]{2}{*}{1450} & \multirow[t]{2}{*}{23} & - & 88 & 0.30 & 6.1 \\
\hline & & & 2 & 50 & 0.225 & 3.1 \\
\hline \multicolumn{7}{|c|}{ Compound of electrodes UONI-13/55 } \\
\hline \multirow[t]{2}{*}{$\mathrm{Na}_{2} \mathrm{O}_{2} \cdot 8 \mathrm{SiO}_{2}$} & \multirow[t]{2}{*}{1430} & \multirow[t]{2}{*}{23} & - & 40 & 0.08 & - \\
\hline & & & 2 & 34 & 0.03 & 5.0 \\
\hline
\end{tabular}


of «rest», when stresses accumulated as a result of specimen strengthening are relaxed [19].

Results of testing of the same on consistency compounds using conical plastometer showed that accumulated by it elastic stresses reduce (following the stop of cone introduction in them) with period of relaxation equal 100-150 s [20]. Complete relaxation of the elastic stresses requires the time significantly exceeding test duration.

Thus, it can be considered that obtained compound indices, even ones being tested in free state, contain significant portion of elastic constituent.

The compounds in course of testing of rheological properties in press chamber of capillary plastometer OB-1435 [9] stay in the conditions of all-round compression, the same on value as in press chamber of industrial electric extrusion unit. Extrusion curves from Figure 1 reflect changes of pressure during pressing-out of the compound from plastometer chamber through lock disk 1 or capillary 2. It can be seen that there are several stages in course of capillary testing:

- stage of development of compound to allround compression in course from beginning of piston movement to moment, when pressure reaches the maximum $\left(P_{\max }\right)$;

- pre-stationary (structural) stage, accompanied by pressure drop (from $P_{\max }$ to $P_{\mathrm{c}}$ );

- setting of pressure of stationary (conditionally equilibrium) capillary flow $P_{\mathrm{c}}$, the value of which is usually used for calculation of $\tau$ and $\theta$;

- pressure drop in the moment of inertia-free stoppage of the piston, caused by free elastic recovery of jet, accompanied by sufficiently quick relaxation of accumulated elastic stresses $\left(\Delta P_{\mathrm{c}}=P_{\mathrm{c}}-P_{\mathrm{ck}}\right)$.

Initial structure of the material is deformed during the first stage, and its elements are oriented and compacted to such extent that so-called structure with limited volume is formed as a result [21]. Considering that this takes place under conditions of virtually zero deformation rates, then huge energy is consumed for such structure formation. Its significant part is accumulated in form of elastic constituent. The material under conditions of all-round compression becomes the elastic body independent of the fact it was solid body or liquid before [14].

The reasons of pressure drop after the maximum lie in growing mechanical and temperature fracture of compound structure, formed in course of previous stage. The center of structure fracture appears in front of enter into the capillary under the effect of pressure gradient and provoked by

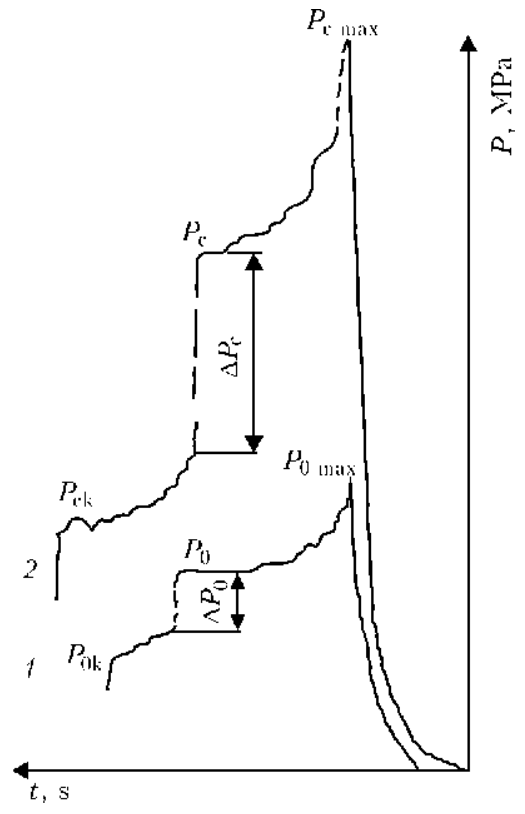

Figure 1. Types of extrusion curves $P=f(t)$ of ANO-4 compound received in its testing by capillary plastometer OB-1435 at $Q=5 \mathrm{~cm}^{3} \cdot \mathrm{s}^{-1}$, diameter of capillary $0.4 \mathrm{~cm}$, length 0 (1) and 4 (2) $\mathrm{cm}$ (for designations see the text)

it asymmetry of potential of molecular interaction between filling agent grains. It facilitates movement of filling agent grains on shear mechanism [21]. The deformation processes, responsible for fracture of the structure, are accumulated in specific moment mainly in the limits of forming natural convergent flow zone. The elastic potential, accumulated in course of the first (growing) branch of pre-stationary process, is a trigger of such energy rearrangement of the structure and transfer of compounds in pressure flow. Fracture of the structure is accompanied by change of relaxation properties of the compound ( $\theta$ reduction).

Rates of fracture and recovery of the structure during equilibrium capillary flow became even on value. However, the system preserves in it significantly larger margin of elastic potential than in studied above tests of open specimens, and it is «relaxed» only after piston stop. Immediate pressure drop is as a rule accompanied by emission of some amount of the compound from shaping head.

Since extrusion takes place under non-isothermal conditions (isothermal ones can not be developed in principle in real rate of compound flowing), then the value of pressure drop, together with compound consistency, should, obviously, be effected by viscous heating, to which the compound is subjected begining from its structural phase. It should also be considered, that liquid glass is a piezo-sensitive liquid [22]. Reduction of its viscosity, caused by excessive pressure, influences the compound structure in the same way as viscous heating. Their properties 
at very high level of compression can change in opposite directions under effect of temperature and pressure. Therefore, the final result is difficult to be predicted.

Results of experimental estimation of viscoelastic characteristics of the compounds under condition of pressure flow. Value of accumulated elastic energy can be estimated on $\Delta P_{0}$ value, if lock disk is used, as in the case of determination of input resistances, or $\Delta P_{c}$, if capillary is applied. The results of estimations are matched on absolute scale $\Delta P_{0}$ and $\Delta P_{\mathrm{c}}$ as well as on relationships $\Delta P_{0} / P_{0}$ and $\Delta P_{\mathrm{c}} / P_{\mathrm{c}}, P_{0}$ $-\Delta P_{0} / P_{0}$ or $P_{\mathrm{c}}-\Delta P_{\mathrm{c}} / P_{\mathrm{c}}$.

Results of testing of the compounds of rutile, low-hydrogen and cellulose types, which were carried out using capillary plastometer OB-1435 at the E.O. Paton Electric Welding Institute in different time, were used for evaluation of visco-elastic indices. Table 3 shows compound characteristic. Compound consistency was regulated by means of change of filling agent composition, its grain composition as well as characteristics of liquid glass. Plastic strength of compounds at that was changed in the ranges from 0.13 (very weak consistency) to $1.40 \mathrm{MPa}$ (structured composition).

Preliminary evaluation of value of pressure drop $\Delta P_{0}$ and $\Delta P_{\mathrm{c}}$ at the moment of piston stop found that the compounds are significantly differ from each other by elastic properties. Portion of elastic constituent in the results of their testing, depending on flow rate and method of its regulation $\left(Q=\right.$ const or $d_{c}=$ const $)$, was changed in proportion to $P_{0}$ and $P_{c}$ value in the ranges from 0 to $60 \%$. It was effected by number of accompanying factors.
For example, notable dissipative heating of the jet was specifically observed in testing of ANO-4 compounds having low heat-conductivity, and UONI-13/55 compounds (ST) manufactured based on liquid glass, which were significantly «fluidized» even under effect of moderate viscous heating. In both cases, effect of dissipative factor dominated at flow mode $Q=$ $=$ const and portion of revealed elastic energy was overstated due to this. In contrast, results of viscous heating in UONI-13/55 (NT) compounds, manufactured based on low-viscosity liquid glasses, were masked by heat consumption used for fracture of their coagulation structure. In this case, the results of tests were understated.

In general, interesting information was received on number of properties of compared compounds, however, they do not allow making single opinion on elastic characteristics.

Table 4 generalizes the results of evaluation of viscous and elastic properties, produced using calculation formula given in Table 1.

Value of rate gradients and, respectively, shear stress, which were used in course of investigations, are limited by ranges, in which studied compounds can be extruded through the nozzles of used sections (consumption per seconds was changed in the ranges from 1 to $2.25 \mathrm{~cm}^{3}$, and $\left.d_{\mathrm{c}}=1-6 \mathrm{~mm}\right)$. Some compounds lock the channels of particularly small diameters, when specific shear rate is exceeded. Shear (as well as longitudinal viscosity) reduces with increase of deformation rate, and this confirms thixotropic fracture of the compounds, forced by their viscous heating to the extent in which they susceptible to it. Longitudinal viscosity 2 times higher than shear one, and relationship $\lambda / \eta$ between them

Table 3. Characteristic of charge, liquid glasses and compounds used

\begin{tabular}{|c|c|c|c|c|c|c|c|}
\hline \multirow{2}{*}{ Compound } & \multirow{2}{*}{ Index } & \multirow{2}{*}{$S_{\mathrm{sp}}, \mathrm{cm}^{-1}$} & \multicolumn{4}{|c|}{ Indices and portion of liquid glass } & \multirow{2}{*}{$P_{m}, \mathrm{MPa}$} \\
\hline & & & Modulus & $\rho, \mathrm{kg} / \mathrm{m}^{3}$ & $\eta, \mathrm{mPa} \cdot \mathrm{s}$ & Portion, \% & \\
\hline \multirow[t]{3}{*}{ ANO-4 } & A2 & \multirow[t]{3}{*}{8300} & \multirow[t]{3}{*}{2.9} & \multirow[t]{3}{*}{1465} & \multirow[t]{3}{*}{800} & 29.6 & 0.30 \\
\hline & A1 & & & & & 29.8 & 0.75 \\
\hline & A4 & & & & & 29.8 & 1.30 \\
\hline \multirow{3}{*}{$\begin{array}{c}\text { UONI-13/55 } \\
\text { (ST) }\end{array}$} & GS-1 & 2250 & \multirow[t]{3}{*}{2.9} & \multirow[t]{3}{*}{1495} & \multirow[t]{3}{*}{1000} & \multirow[t]{3}{*}{25.0} & 0.15 \\
\hline & GS-4 & 3900 & & & & & 0.13 \\
\hline & GS-6 & 4900 & & & & & 0.28 \\
\hline \multirow{3}{*}{$\begin{array}{c}\text { UONI-13/55 } \\
(\mathrm{NT})\end{array}$} & GS-1 & 2250 & \multirow[t]{3}{*}{3.2} & \multirow[t]{3}{*}{1334} & \multirow[t]{3}{*}{50} & \multirow[t]{3}{*}{22.0} & 0.45 \\
\hline & GS-4 & 3900 & & & & & 0.30 \\
\hline & GS-6 & 4900 & & & & & 0.80 \\
\hline VSTs-4 & Ts & - & $2.9^{*}$ & 1407 & 100 & 51.5 & - \\
\hline
\end{tabular}


Table 4. Indices of viscous elasticity of electrode compounds (capillary zone)

\begin{tabular}{|c|c|c|c|c|c|c|c|c|c|}
\hline \multirow{2}{*}{ Compound } & \multirow[b]{2}{*}{ Indices } & \multirow{2}{*}{$\gamma, \mathrm{s}^{-1}$} & \multicolumn{7}{|c|}{ Value of indices of viscous elasticity } \\
\hline & & & $\tau, \mathrm{MPa}$ & $\eta, \mathrm{MPa} \cdot \mathrm{s}$ & $G, \mathrm{MPa}$ & $\theta, \mathrm{s}$ & $\begin{array}{c}W, \\
\mathrm{MPa} / \mathrm{cm}^{3}\end{array}$ & $\theta \dot{\gamma}$ & $P_{0}, \mathrm{MPa}$ \\
\hline \multirow[t]{6}{*}{ ANO-4 } & A2 & 11.8 & 0.43 & $\underline{0.000470}$ & 0.018 & $\underline{0.014}$ & 0.83 & 19.4 & 10.0 \\
\hline & & 1445 & $\overline{0.70}$ & $\overline{0.039090}$ & $\overline{0.035}$ & $\overline{2.110}$ & $\overline{1.92}$ & $\overline{33.3}$ & $\overline{23.0}$ \\
\hline & A1 & 11.8 & 0.61 & 0.008900 & 0.022 & 0.026 & 1.23 & 22.3 & 15.5 \\
\hline & & $\overline{1211}$ & $\overline{1.10}$ & 0.055890 & $\overline{0.034}$ & $\overline{2.320}$ & $\overline{2.83}$ & $\overline{32.6}$ & $\overline{34.0}$ \\
\hline & A4 & 11.8 & 0.78 & $\underline{0.000860}$ & 0.020 & 0.040 & 1.96 & 11.7 & 23.5 \\
\hline & & $\overline{1211}$ & $\overline{1.19}$ & 0.025785 & 0.045 & $\overline{2.830}$ & 4.10 & 48.5 & 49.0 \\
\hline \multirow{6}{*}{$\begin{array}{c}\text { UONI-13/55 } \\
\text { (ST) }\end{array}$} & GS-1 & 11.8 & 0.50 & $\underline{0.000950}$ & $\underline{0.017}$ & $\underline{0.010}$ & 1.15 & 11.8 & 14.0 \\
\hline & & $\overline{1650}$ & $\overline{1.55}$ & 0.041113 & 0.085 & $\overline{2.450}$ & $\overline{2.75}$ & $\overline{31.7}$ & $\overline{33.0}$ \\
\hline & GS-4 & 11.8 & 0.70 & 0.000550 & 0.038 & 0.018 & 1.15 & 14.8 & 10.5 \\
\hline & & $\overline{1650}$ & $\overline{1.40}$ & $\overline{0.060170}$ & $\overline{0.065}$ & $\overline{1.255}$ & $\overline{2.55}$ & $\overline{21.3}$ & $\overline{42.0}$ \\
\hline & GS-6 & 11.8 & 0.80 & $\underline{0.000080}$ & $\underline{0.028}$ & $\underline{0.008}$ & 1.15 & 16.8 & 13.8 \\
\hline & & 1650 & 1.25 & 0.069500 & 0.075 & 1.425 & 2.50 & 38.5 & 90.2 \\
\hline \multirow{6}{*}{$\begin{array}{c}\text { UONI-13/55 } \\
(\mathrm{NT})\end{array}$} & GS-1 & 11.8 & 0.31 & 0.000090 & $\underline{0.005}$ & 0.005 & 1.25 & 34.8 & 15.3 \\
\hline & & $\overline{8120}$ & $\overline{0.75}$ & $\overline{0.026270}$ & $\overline{0.019}$ & $\overline{4.370}$ & $\overline{2.50}$ & $\overline{61.8}$ & $\overline{30.0}$ \\
\hline & GS-4 & 11.8 & $\underline{0.25}$ & $\underline{0.000010}$ & $\underline{0.003}$ & $\underline{0.001}$ & 1.28 & $\underline{25.0}$ & 15.9 \\
\hline & & $\overline{64970}$ & $\overline{0.75}$ & $\overline{0.033900}$ & $\overline{0.023}$ & $\overline{3.390}$ & $\overline{2.50}$ & $\overline{86.0}$ & $\overline{37.2}$ \\
\hline & GS-6 & 11.8 & 0.40 & 0.000015 & 0.007 & 0.001 & 1.35 & 26.0 & 19.8 \\
\hline & & $\overline{64970}$ & $\overline{0.95}$ & 0.037290 & 0.027 & $\overline{3.720}$ & 2.25 & $\overline{65.0}$ & $\overline{33.0}$ \\
\hline \multirow[t]{2}{*}{ VSTs-4 } & Ts & 150.0 & $\underline{0.38}$ & 0.000005 & $\underline{0.005}$ & 0.001 & 0.90 & 20.9 & 11.0 \\
\hline & & $\overline{64970}$ & $\overline{0.60}$ & $\overline{0.003490}$ & $\overline{0.025}$ & $\overline{0.140}$ & $\overline{3.10}$ & $\overline{65.0}$ & $\overline{37.0}$ \\
\hline
\end{tabular}

rises with increase of shear rate, that promotes decrease of angle of natural convergence, or reduction, but at that rise of $\alpha_{0}$. Small $\alpha_{0}$ value indirectly indicate that compound jet in pre-capillary zone overcomes resistance of the material with high elasticity. Modulus of elasticity under these conditions also shows ambiguous change.

Its value in all ANO-4 compounds does not depend on their consistency and shear rate.

Modulus of elasticity for compounds UONI$13 / 55$ (ST) significantly increases with rise of shear rate. It is initially the lowers one on value for GS-1 variant with the coarsest filling agent. The finer filling agent grains, the higher is the modulus of elasticity for this type of compounds at low shear rates, and it is lower at high shear rates. The weakest reaction on deformation rate was observed in compound GS-6 with the most pronounced structure. Grain composition of the filling agent has the similar effect on modulus of elasticity of UONI-13/55 (NT) compound with low-viscosity liquid glass. However, value of their modulus of elasticity, is initially smaller on value than in $\mathrm{CT}$ compounds and has weaker response to deformation rate. The compound with average grain-size of the filling agent showed significant scatter of results. The reason of this is unknown.
Modulus of elasticity in VSTs-4 compound reduces with rise of shear rate gradient.

Generally, it should be noted that average value of elasticity modulus of compound changed in sufficiently narrow limits regardless the wide range of change of consistency of compared compounds.

There was no information in mastering of procedure of evaluation of compound elasticity on actual value of their modulus of elasticity, except for data of V.I. Klementov, received in mode of creep flow. In this connection, predicted order of value of this index was calculated in the following way.

It is well know fact that one of the effects, accompanying free recovery of jet of visco-elastic compositions, is its swelling at output from the capillary (Barrus-effect). Technologies of polymer processing, characterized by pronounced non-Newtonian properties, can have relationship of jet diameter and extrusion nozzle achieving 4 -fold value. Introduction of filling agents in the polymers together with reduction of price of products provide for suppression of this undesirable effect. Electrode compounds are also filled compositions with liquid-glass matrix, having weak non-Newtonian properties. There should be 
no swelling of jet in such compositions by this reason. It is indicated by good matching of coating diameters (jet, when it is a question of compound extrusion at capillary testing) and extrusion nozzle (capillary).

In fact, insignificant swelling of the compound jet still takes place. It is determined that rearrangement of rate profile in the jet takes place at the output from capillary in hydraulic and rheology, as a result of what, its diameter should decrease at least by $13 \%$ based on law of momentum conservation.

In other words, elastic swelling of the compound jet as though stays in $\beta=0.13$ limits, compensating its constriction, promoted by rearrangement of rate profile.

If $\beta=0.13$ in equitation (9) is substituted, then swelling-caused deformation will make $\gamma=$ $=18.5 \%$. On the other hand, it was taken into account that $\gamma$ and $G$ are related with each other by $\gamma=\tau G$ relationship [8].

As a rule, shear stress on the capillary wall in compounds varies in the ranges from 0.5 to 1.5 MPa. It should be expected based on this that value of elasticity modulus of the electrode compounds lies in the ranges from 0.025 to $0.075 \mathrm{MPa}$. According to order of value it agrees with our results, given in Table 4, and results of V.I. Klimentov (see Table 2).

Change of elastic potential and input resistances are symbate to each other in rise of shear rate. This verifies that elastic state of the com-

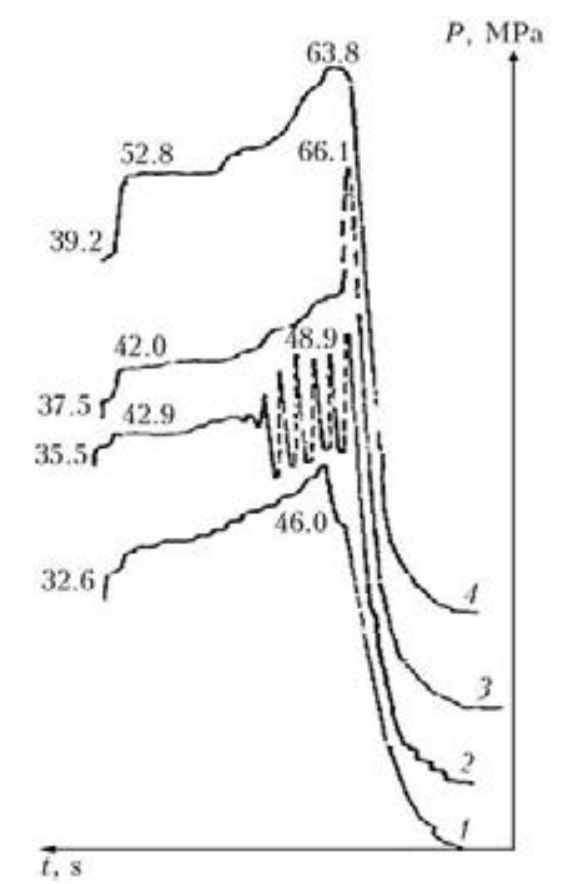

Figure 2. Extrusion curves of compounds GS-6 (NT) at $Q=$ $=1 \mathrm{~cm}^{3} \cdot \mathrm{s}^{-1}$ and capillary diameter of $6(1), 4(2), 2$ (3) and 1 (4) $\mathrm{mm}$ pounds takes place in the convergent zone. Its relaxation manly appears out of its limits.

Period of relaxation of studied compounds at capillary stage in logarithmic interpretation linearly reduces with rise of deformation rate. This indicates dispersal of the elastic energy, accumulated in the pre-capillary zone, due to mechanical and temperature fracture of the compound structure. Level of viscosity exceeds the elasticity modulus at small shear rates, and becomes smaller than at high ones. Zero transition point is easy to determine, assuming that viscosity and modulus of elasticity become equal in value at $\theta=1$. In this moment any accidental influence on the system can promote different nature oscillations of the compound flow rate under the effect of stress elastic constituent, since viscosity of the extruded material can not already suppress them. Most often pulsations appear in the stage of incident (structural) branch of the extrusion diagram. Figure 2 as example shows pulsation curve registered in extrusion of GS-6 (NT) compound through $4 \mathrm{~mm}$ diameter capillary at $\dot{\gamma}=$ $=40 \mathrm{~s}^{-1}$ rate. Nature of pulsation allows assuming that detachments of the jet take place on the capillary wall. Other variants of compounds of GS series tested in parallel show no pulsation.

Two reasons can explain this. The first is related with step-like nature of change of mode of compound extrusion in plastometer OB-1435. As a result, only experiment with compound GS-6 (NT) provides the extrusion conditions suitable for critical period of relaxation $\theta=1$, at which pulsation of pressure flow can take place. Measurements of the rest experiments were out of such critical point. The second reason is connected with the fact that flow pulsation in the capillary zone takes place on channel surface. Decrease of capillary diameter promotes rise of flow rate, reduction of material viscosity and, seemingly, conditions for pulsing improve at relatively stable value of elasticity modulus. In reality, reduction of channel diameter results in simultaneous rise of specific surface of nozzle (in calculation per unit of volume) and its suppressive action on the jet. It is known from theory of near-wall slipping that it mostly appears in using of wide channels and being suppressed in their replacing by small diameter capillary.

Figure 3 shows the diagrams of extrusion of the same compounds GS-1, GS-4 and GS-6, made based on low-viscosity glasses, through capillary of $4 \mathrm{~mm}$ diameter with movable insert («vane») in front of capillary input. It was designed for provoking of non-stationary flow. It was found 
that flows of the compounds with coarse- (SG-1) and average-grain (GS-4) filling agents showed no reaction on flexible insert before input into the shaping channel. Indicated insert in the case of GS-6 compound with fine-grain filling agent provoked sufficiently continuous gradually attenuating pulsation of the flow. This experience proves that tendency to non-stationary flow modes is, first of all, a compound property and only then being a condition of its pressure flow. Pulsation modes of compound flow can be developed not only on the capillary wall, but in the convergent zone as well. And conditions for their appearance in the convergent zone are more favorable than on the capillary wall.

It is confirmed by results of investigation of pilot variants of UONI-13/55 compound, marked by T-9 index. The compound is made on three-module liquid glass with $850 \mathrm{mPa} \cdot \mathrm{s}$ viscosity. Share of liquid glass in it makes $26 \%$. Characteristic feature of the filling agent is its grain composition. It in accordance with the experiment purposes has the following share proportion of fractions, i.e. total residual on meshes of 250 , 160,100 and $63 \mu \mathrm{m}$ made respectively $6,8,13$ and 37 vol.\%. Specific surface of charge $S_{\mathrm{sp}}=$ $=10,000 \mathrm{~cm}^{-1}$, level of volume filling by particles makes $F_{m}=0.67$. Consistency of compound is sufficiently tight, thus $P_{m}=0.58 \mathrm{MPa}(0.85 \mathrm{MPa}$ after hour holding). Results of experiments are given in Figure 4.

It was determined in process of extrusion tests that pressure of compound flow through lock disks with hole diameters 6,4 and $2 \mathrm{~mm}$ made 50,55 and $57 \mathrm{MPa}$. The figures themselves are not very high. The compound passed through $6 \mathrm{~mm}$ diameter hole with sufficiently small pulsations. Flowing through 4 and $2 \mathrm{~mm}$ diameter holes was accompanied by significant rate pulsations. The first of them reflects the flow at $40 \mathrm{~s}^{-1}$ shear rate gradient, the same as in experiment with GS-6 compound in capillary of the same diameter. Therefore, the first experiment with T-9 compound also reproduced the critical conditions ( $\eta$ and $G$ ), under which pulsations of flow rate take place, in this case in the entrance zone. Extrusion of T-9 compound through $2 \mathrm{~mm}$ diameter hole in disk takes place at $318 \mathrm{~s}^{-1}$ rate, i.e. 8 times higher than in previous case. At that shear viscosity of the compound should significantly reduce, and modulus has to rise. The conditions for flow pulsation have become more favorable, and shape of extrusion curve verifies this. In fact, flow pulsation, caused by excess of the compound elasticity, is already suppressed no by viscosity, neither by capillary limiting sur-

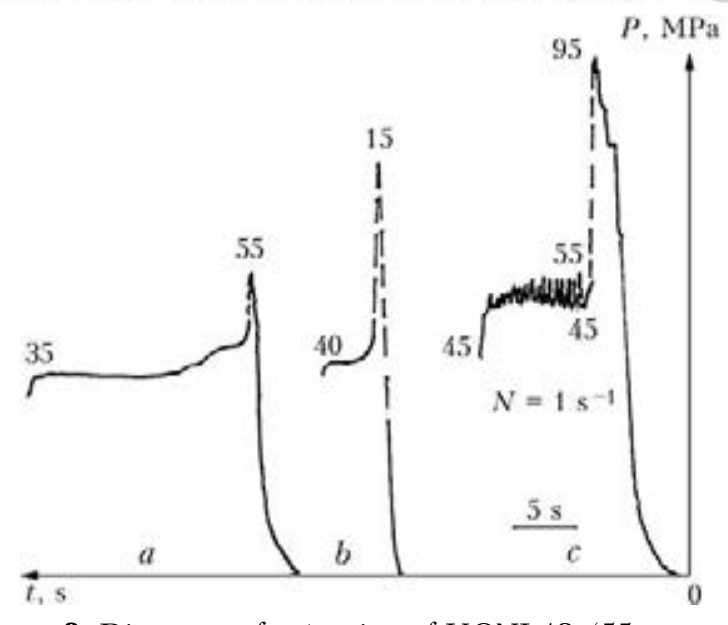

Figure 3. Diagrams of extrusion of UONI-13/55 compound manufactured based on high-modulus liquid glass with viscosity $100 \mathrm{mPa} \cdot \mathrm{s}$ at $Q=1 \mathrm{~cm}^{3} \cdot \mathrm{s}^{-1}$, conical nozzle $\left(2 \alpha=40^{\circ}\right.$, $4 / 40 \mathrm{~mm}$ diameter) and portion of fine fraction of $20(a)$, $40(b)$ and $60(c) \%$

face, since compound flow in the convergent zone takes place over shear layers. Shape of peaks is smoother in contrast to sharp peaks in the case of GS-6 compound, which slipped over capillary surface.

Immediate relaxation of accumulated elastic stresses in the convergent zone is often observed in form of pulsing outcomes of the compound from shaping channels of extrusion head under real conditions of electrode extrusion. For example, if extrusion head has two-channel guide, compounds with excessive elasticity can by turn select one of the channels for flowing, whereas its flow in parallel channel of the same profile is stopped for this time. Flow pattern in the next phase of the process is changed to the opposite, i.e. compound chooses for passing the channel,

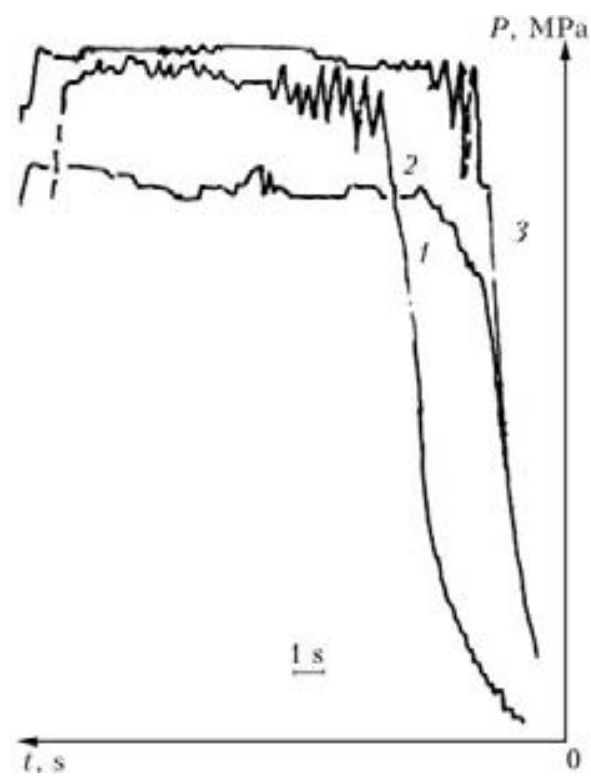

Figure 4. Comparison of extrusion curves $P_{0}=f(t)$ of T-9 compound, produced with the help of capillary viscosimeter, at nozzle diameter of 2 (1), 6 (2) and 4 (3) mm 
in which it was stationary up to this moment. Compound flow in the parallel channel is stopped for the same period time.

Some results of investigation of regularities of thickness difference appearance under real conditions of electrode extrusion. Thickness difference of the coating appear in bi-component flow coming from coating to rod, as a result of stochastic on its nature energy interaction of the components, one of which (the coating) is characterized by non-linear visco-elastic properties, susceptible to appear in form of pulsating flows, and another (the rod) is elastic element. Relation of viscosity and elasticity in coating material to greater or lesser degree is changed under the effect of dissipative and mode flow factors, and accompanied by breaking of its stability, whereas elastic properties of the rod remain unchanged at that. Role of the effects, related with elastic turbulence, is characterized by $\operatorname{Re}_{\mathrm{e}}=U t^{2} / \theta R$ criterion. Effects of interruption of flow stability, appearing in turning of compound flow, in dividers and conical channels before entering into the shaping head, as well as in area of hydrodynamic stabilizing of flow at input of the extrusion nozzle, can be characterized by monochromatism criterion $H_{0}=U t / R$ [15].

Stationary or attenuating, regular or irregular oscillations of the compound rate, accompanying by pulsation of rate and pressure, provoke alternating transverse deviations of the rod from axis of the calibration insert. As a result, significant
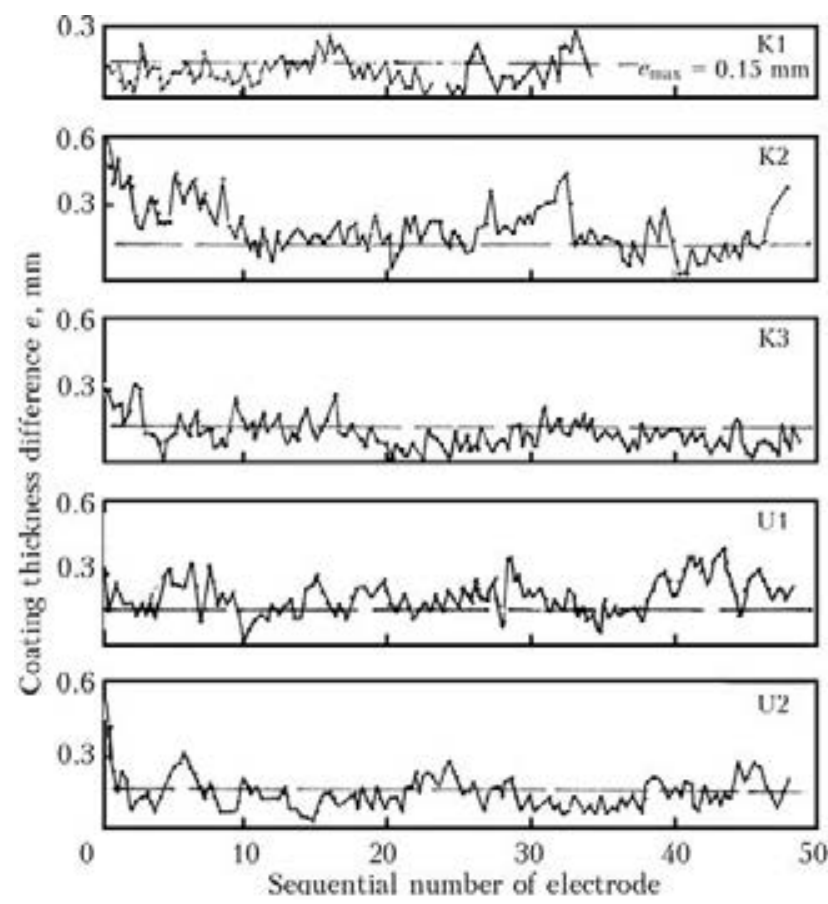

Figure 5. Dynamics of change of coating thickness difference during extrusion of pilot low-hydrogen electrodes of $3 \mathrm{~mm}$ diameter with index K1-K3, U1 and U2 distortion of uniform on section circular shape of the coating takes place. Combination of transverse oscillations with longitudinal movement of the rod can result in oscillation as well as helical change of position of maximum value of thickness difference along the electrode.

Eccentric position of coating cross section relatively to the rod does not change its area in comparison with its concentric shape. However, many liquids experience lower resistance in their flowing through the eccentric channels in comparisons with the channels of concentric shape. Work [23] determined this during investigation of pressure flow of water solutions KMTs, GETs and MTs through the circular channels between coaxial pipes. It was found that pressure falls, necessary for their pressure flow through the eccentric channels at fixed flow rate, reduce with rise of eccentricity. The liquids with more pronounced non-Newtonian properties show slower rate of pressure drop reduction depending on rise of value of eccentricity between external and internal pipe. Such a flow pattern can be used during circular extrusion of rod casing, replacing surface of internal pipe to rod surface, moving synchronously with compound.

Monitoring of dynamics of thickness difference change show that it reflects well the effect on this index of found by us peculiarities of viscoelastic behavior of compounds.

Figure 5 gives the results of testing of five types of compounds for low-hydrogen electrodes different in technological properties. Thickness difference of the coating was measured at the beginning, middle and end of each electrode, came out from head of the press. Extrusion of electrodes with $3 \mathrm{~mm}$ diameter rod was carried out on the Oerlikon straight-flow press EP-120. It can be seen that more or less pronounced initial period can be, first of all, outlined in change of thickness difference of the coating during application of each of tested compounds over the rods. In course of this period the compound passes from condition of the most compression into relatively stationary flow mode. At that, larger on value initial thickness difference of the coating reduces and gradually reaches the level, which varies with respect to some average oscillating value. Waves of these oscillations differ on amplitude and frequency, at that drop and rise of low-frequency wave is also accompanied by finer on amplitude, but more often (even in the limits of one electrode) oscillations of thickness difference. It indicates its random nature, reflecting very complex changes in relationship of viscous and elastic characteristics of the compounds un- 

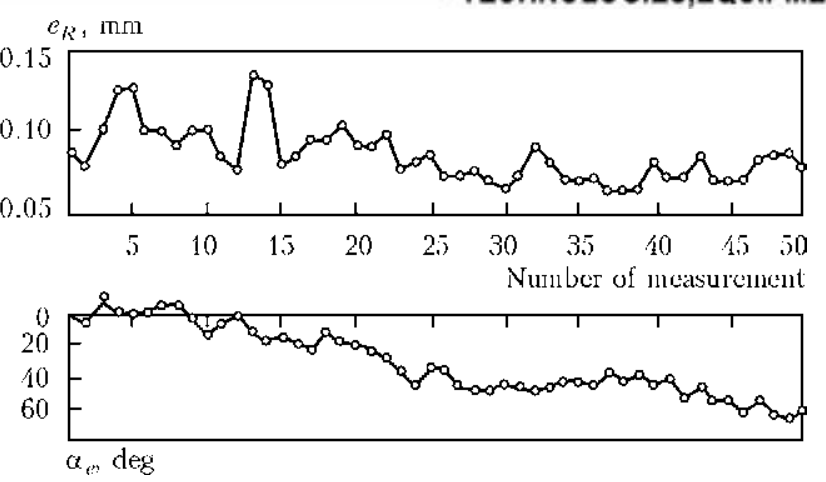

Figure 6. Change of value and angle of orientation of vector of coating thickness difference on oscillography data

der condition of pressure flow with rod. Given examples do not show the cases of thickness difference, reason of which could be explained by entering of some random inclusions in the compound.

Figure 6 gives data on value and angle of orientation (in plane normal to electrode axis) of vector of coating thickness difference. They were obtained by oscillographic measurements of its horizontal and vertical constituents in the course of production of UONI-13/55 electrode with $4 \mathrm{~mm}$ diameter rod using the Havelock straightthrough press [24].

200 electrodes were produced by head of the press during monitoring, value of thickness difference vector $e_{R}$ changed from $0.15-0.17$ to $0.07-0.10 \mathrm{~mm}$ (i.e. almost 2 times) and angle of its orientation with respect to $\alpha_{e}$ horizont rised from 0 up to $60^{\circ}$. Pulsation of $e_{R}$ and $\alpha_{e}$ was observed against a background of their general changes. Vertical constituent of thickness difference shows specific pulsation and it is explained by vertical positioning of two windows of diaphragm-divider, through which the compound is pumped from cylinder to press chamber (gap between tip of rod-guide and calibrating insert). Figure 7 shows relationship of value and angle of orientation of vector of thickness difference. The lager its deviation from horizontal line, the smaller is the amplitude of its oscillations. However, its absolute value at that increases. It seems that there are wave-like asynchronous oscillations of $e_{R}$ and $\alpha_{e}$ (the first are faster in time than the second), thus some kind of spatial (spiral) wave is formed. In one direction $e_{R}$ reduces with $\alpha_{e}$ rise and in other, normal to the first, it increases with the same increments of $e_{R}$ and $\alpha_{e}$. It is supposed that flow oscillations, caused by its $90^{\circ}$ horizontal turning at the output from cylinder in front of the divider, are superimposed over compound pulsation through the divider windows. It can be assumed that in this case internal and external surfaces should move at

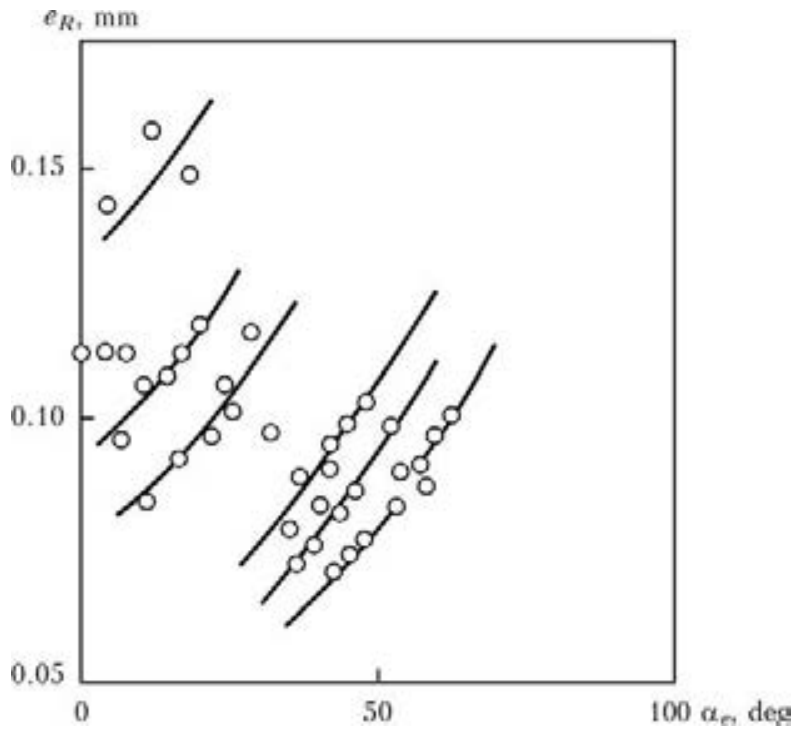

Figure 7. Relationship of value and angle of orientation of vector of coating thickness difference found by oscillography

different rates due to necessity of fulfillment of principle of compound flow homochronicity (continuity). The closer to the horizon the positioning of thickness difference vector, the greater is the effect of this factor on thickness difference.

Boundaries and levels of randomness of deviation of coating thickness difference on its main orientation can be judged on results of measurements, carried out using concentricity meter KRP-12 during extrusion of ANO-4 electrodes with $4 \mathrm{~mm}$ diameter rod at angle press MAOE-1.

Vertical press has compound flow turning angle $90^{\circ}$. «Windows» of the divider of compound flow are vertically oriented in head of the press.

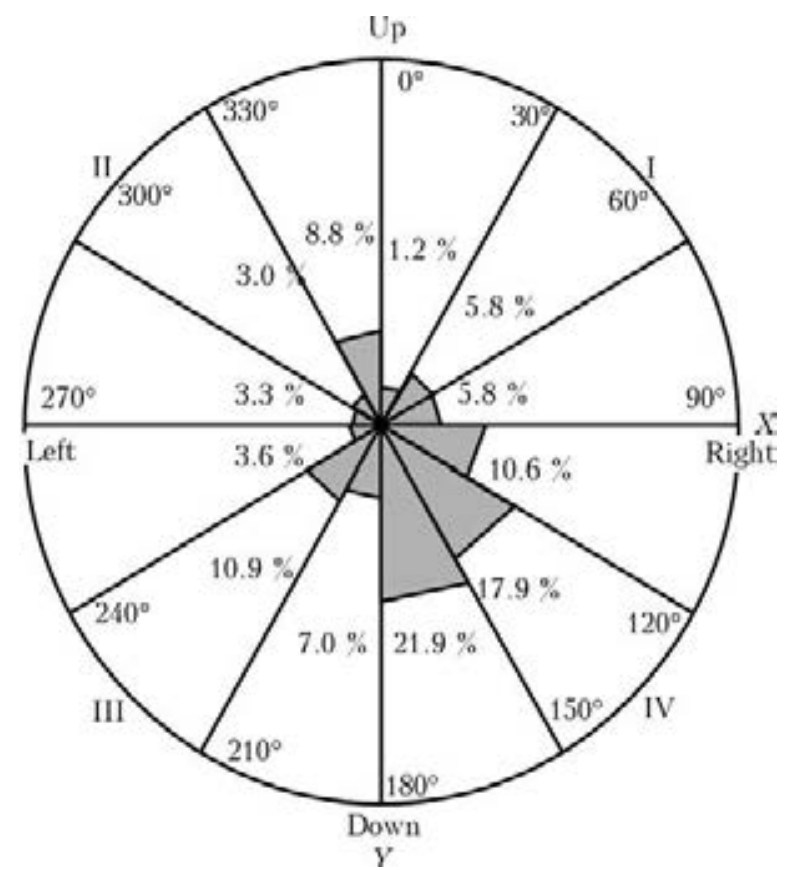

Figure 8. Statistical distribution of angle of orientation of vector of coating thickness difference in plane normal to axis of coming electrodes for 980 electrodes in sampling 
TECHNOLOGIES,EQUIPMENT AND CONTROL IN CONSUMABLES PRODUCTION

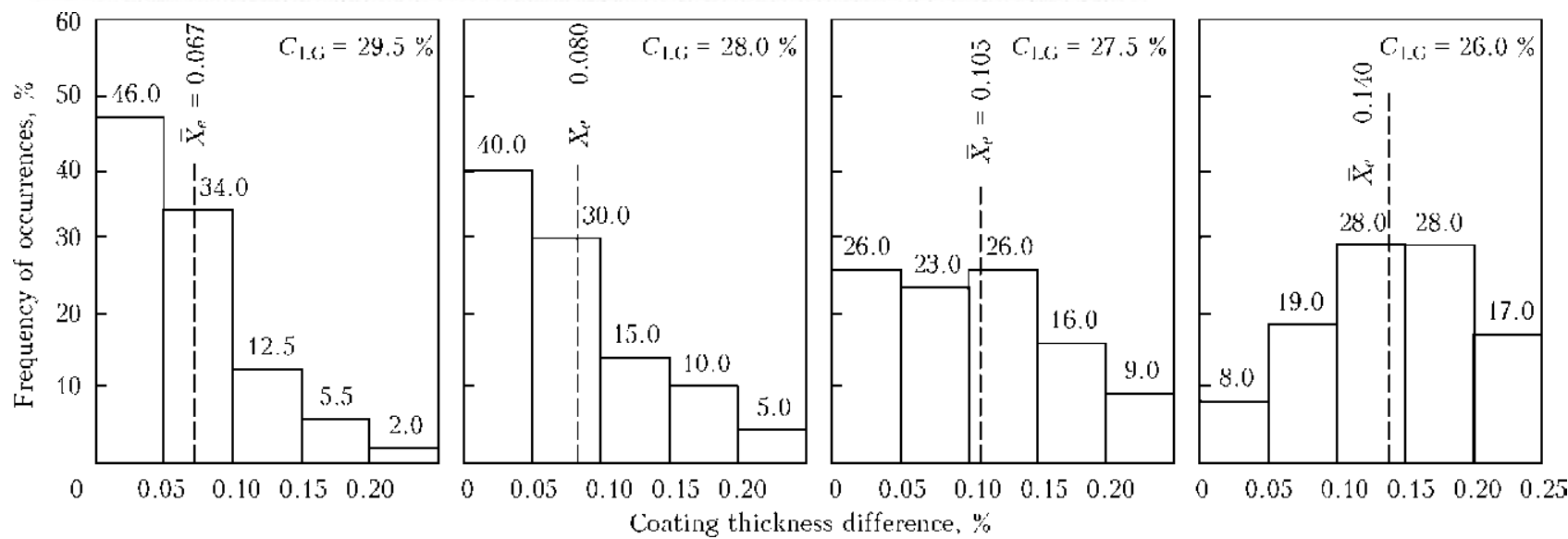

Figure 9. Effect of portion of liquid glass $C$ in compound on tendency of ANO-4 electrodes to coating thickness difference

Solid line, plotted as generatrix in zenith position of coating of the electrodes produced by press, was a reference point for angle $\alpha_{e}$. During measurement the second reference mark, corresponding to the position of maximum thickness difference, was plotted in each of 11 control sections of the electrode. Angle between these marks in plane normal to the electrode axis was taken as $e_{R}$ orientation angle. Frequency distribution of measurement results in earlier selected sectors is shown in Figure 8. This shows that the maximum values of coating thickness difference are oriented mainly in the sectors between 120 and $180^{\circ}$. Shifting of the rod, being the reason of coating thickness difference, is manly promoted by external side of compound flow upward and to the left relatively to vertical line.

Figure 9 represents the results of estimation of coating thickness difference of the electrodes, portion of liquid glass in which was changed from 29.5 to 26.0 wt. $\%$.

As far as consistency of the compound became more and more elastic, share of electrodes with excessive coating thickness difference increased from 2 to $17 \%$. Effect of increased portion of elastic constituent promoted rise of average value of thickness difference from 0.067 to $0.140 \mathrm{~mm}$. Shape and width of static scattering of this index (from one-sided to Gaussian) were changed.

\section{Conclusions}

1. Coating thickness difference takes place in bi-component flow, in which casing from compound is characterized by non-linear visco-elstic properties and rod is the component with constant elasticity modulus. Relationship of viscosity and elasticity in the casing material reduces under the effect of structure fracture and dissipative heating, and this, as a result, provokes instability of shear deformation modes, and so, can be the reason of violation of uniform coating application over the rods.

2. It is determined that mathematical apparatus, developed in the polymer rheology, can be used for calculation of such visco-elastic characteristics of the electrode compounds under condition of capillary flow as relaxation period, modulus of elasticity, elastic potential and Reynolds criterion of elastic turbulence.

3. Using of indicated apparatus allows calculation of listed indices of visco-elastic electrode compounds for rutile, low-hydrogen and cellulose electrodes, consistency of which was regulated in sufficiently wide limits by change of grain compositions of charge, characteristics of liquid glass and its portion in electrode compound. On the other hand, changing of modes of capillary testing of indicated compounds allows determining their effect on rheological properties.

4. It is determined that modes of compound pressure flow provide for the largest effect on shear viscosity and period of compound relaxation, whereas their elasticity modulus is significantly less effected. Thus, 4-5 order rise of gradient of shear rate promoted 4 orders reduction of viscosity and relaxation of compounds. It is caused by mechanical and dissipative fracture of compound coagulation structure. Modulus of elasticity under the same conditions of testing is constant for many compounds (all types of rutile and low-hydrogen compounds with fine-grain filling agent), it rises not more than by order (low-hydrogen compounds with coarse-grain filling agent, manufactured based on viscous-liquid glass) or reduces to insignificant level (cellulose compounds).

5. Slope opposition of change of viscosity and period of relaxation, on the one hand, and modulus of elasticity, on the other hand, suggest that viscosity and modulus of elasticity become even 
on value at determined rate, and period of relaxation, which is a relationship of viscosity and modulus of elasticity, become equal one. After this, viscosity loses the possibility to damp elastic oscillations of the compounds, which can be caused by any random reasons, and system passes in mode of unsteady flow, expressed with different level of regularity.

6 . The work shows two types of unsteady flow, namely in capillary and entrance (pre-capillary) zone. In the first case, it has features of jet blowing out on capillary wall and it can be suppressed by using of smaller section capillaries, that is typical for near-wall slipping effect. In the second case, unsteady flow mode does not disappear at transfer to lock disk with hole of smaller section. Therefore, in this case, unfavorable relationship of viscosity and elasticity is preserved in rise of shear rate. Introduction of soft elements, capable to promote unsteady flow, works only with those compounds, which can pulsate without flexible insert. Thus, tendency to unsteady flow modes, capable to result in coating thickness difference, and external disturbing factors only reveal this capability.

7. Qualitative dependence of elastic turbulence of electrode compounds on nature and character of appearance of coating thickness difference under real extrusion application of coating over rod electrodes is shown.

1. Marchenko, A.E. (2000) Coating thickness difference as a factor of process state and quality of manufacture of welding electrodes. In: Proc. of Sci.-Techn. Seminar on Electrode Manufacturing on the Threshold of New Millennium (St.-Petersburg, 22-26 May 2000), 124-125. Cherepovets: Elektrod.

2. Vornovitsky, I.N. (1989) Coating thickness difference as a main factor of quality of electrodes. Svarochn. Proizvodstvo, 4, 7-19.

3. Mayasaka, K., Oshiba, F., Akamatsu, T. (1982) Effect eccentricity of the coating upon the deposition of weld metal. J. JWS, 7, 47-55.

4. Ovchinnikov, V.A., Bazhenov, V.V. (1978) Influence of electrode coating geometry and presence of defects in it on reliability of melted metal shielding from air action. Svarochn. Proizvodstvo, 5, 39-40.

5. Pokhodnya, I.K., Makarenko, V.D., Milichenko, S.S. (1985) Influence of coating eccentricity on welding-technological properties of electrodes and quality of deposited metal. Avtomatich. Svarka, 11, 20-22.

6. Stepanosov, A.R. (1989) Expert judgement of causes of coating thickness difference of welding electrode. Svarochn. Proizvodstvo, 4, 7-9.

7. Belkin, I.M., Vinogradov, G.V., Leonov, A.I. (1967) Rotary devices. Measurement of viscosity and physico-mechanical characteristics of materials. Moscow: Mashinostroenie.

8. Vinogradov, G.V., Malkin, A.Ya. (1977) Rheology of polymers. Moscow: Khimiya.

9. Marchenko, A.E., Gnatenko, M.F. (1980) Peculiarities of flow of electrode compounds detected by capillary plastometer: SMEA Inform. Doc., Issue 1, 106-117. Kiev: Naukova Dumka

10. Bagley, E.B. (1957) End correction in the capillary flow of polyethylene. J. Appl. Phys., 28(5), 624-627.

11. Cogswell, F.N. (1972) Converging flow of polymer melts in extrusion dies. Polymer Eng. and Sci., 12(2), 64-70

12. Marchenko, A.E. (2011) About rheological properties of electrode compounds in convergent zone during electrode extrusion. In: Proc. of 6th Int. Conf. on Welding Consumables. Development. Technology. Manufacture. Quality. Competitiveness (Krasnodar, 6-9 June 2011), 223-232.

13. Shoff, R.N., Cancio, L.V., Chida, M. (1977) Extrusial flow of polymer melts. Transact. of Soc. of Rheology, 21(3), 429-434.

14. Khan, Ch.D. (1979) Rheology in processes of recycling of polymers. Moscow: Khimiya.

15. Malkin, A.Ya., Leonov, A.I. (1963) On criteria of instability of shear deformation conditions of elasticviscous polymer systems. Doklady AN SSSR, 151(2), 380-383.

16. Philippoff, W., Gaskins, F.H. (1958) The capillary experiment in rheology. Transact. of Soc. of Rheology, 263-284.

17. Sokolov, E.V. (1950) Electrodes with high quality coating and their production. Avtogen. Delo, 11, 26-29.

18. Klementov, V.I. (1953) Liquid glass as a material of electrode coatings for electric arc welding: Syn. of Thesis for Cand. of Techn. Sci. Degree. Moscow.

19. Marchenko, A.E., Skorina, N.V. et al. (1972) Stateof-the-art and prospects of improvement of technology and equipment for manufacture of welding electrodes. In: Proc. of All-Union Conf. on Welding Consumables (Kiev, 31 Oct. -3 Nov. 1972), Pt 2, 210-250.

20. Marchenko, A.E., Shkurko, S.A. (1973) Control of electrode compounds by conic plastometer. In: Proc. of Short-Term Seminar on Electrodes and Fluxes for Electric Arc Welding (Leningrad, 12-14 March 1973), 22-32.

21. Bibik, E.E. (1981) Rheology of dispersion systems. Leningrad: LGU.

22. Marchenko, A.E. Skorina, N.V., Sidlin, Z.A. et al. (1992) Investigation of viscosity of liquid glasses under electrode extrusion pressure. In: New Welding and Surfacing Materials and their Application in Industry: Proc. of Sci.-Techn. Seminar Dedicated to 100th Anniversary of K.V. Petranya (St.-Petersburg, 19-20 May 1992), 43-49.

23. Mitsubishi, N., Aoyagi, Y. (1973) Non-newtonian fluid flow in an eccentric annulus. J. Chem. Eng. of Japan, 6(5), 402-408.

24. Marchenko, A.E., Gnatenko, M.F. (1992) Peculiarities of coating thickness difference formation detected by oscillography In: Abstr. of Sci.-Techn. Conf. on Metallurgy of Welding and Welding Consumables (St.-Petersburg, 1-2 June 1992), 98-100.

Received 22.04.2014 